\title{
Behavioral and brain mechanisms mediating conditioned flight behavior in rats
}

Michael S. Totty ${ }^{1}$, Naomi Warren ${ }^{1}$, Isabella Huddleston ${ }^{1}$, Karthik R. Ramanathan ${ }^{1}$, Reed L. University, College Station, TX, 77843-3474, USA

${ }^{2}$ Corresponding author

Email: maren@tamu.edu

Phone: 979-458-7960

24 Address: Stephen Maren

Department of Psychological and Brain Sciences

26 Texas A\&M University

27301 Old Main Dr.

28 College Station, TX 77843-3474

$\underline{O R C i D}$

Michael S. Totty:

https://orcid.org/0000-0002-9292-8556

Karthik R. Ramanathan: https://orcid.org/0000-0002-8843-3665

Reed L. Ressler:

https://orcid.org/0000-0003-0514-8269

https://orcid.org/0000-0002-9342-7411 


\section{ABSTRACT}

39 Environmental contexts and associative learning can inform animals of potential threats, though it

40 is currently unknown how contexts bias defensive transitions. Here we investigated context-

41 dependent flight responses in the Pavlovian serial-compound stimulus (SCS) paradigm. We show

42 here that SCS-evoked flight behavior in male and female rats is dependent on contextual fear.

43 Flight was reduced in the conditioning context after context extinction and could be evoked in a

44 different shock-associated context. Although flight was exclusive to white noise stimuli, it was

45 nonetheless associative insofar as rats that received an equal number of unpaired USs did not show

46 flight-like behavior. Finally, we found that inactivation of either the central nucleus of the

47 amygdala (CeA) or bed nucleus of the stria terminalis (BNST) attenuated both contextual fear and

48 flight responses. This work demonstrates that contextual fear summates with cued and innate fear

49 to drive a high fear state and freeze-to-flight transitions. 


\section{INTRODUCTION}

62 The selection of appropriate defensive behavior is vital to survival in the face of threat. Associative

63 learning allows animals and humans to adapt their behavior to avoid predicted danger, and

64 environmental contexts are critical for discriminating between fear and safety. Traumatic events

65 can lead to pathological fear and the dysregulation of contextual processing appears to be central

66 to various psychopathologies, such as post-traumatic stress disorder (PTSD) (Liberzon and

67 Abelson, 2016; Liberzon and Sripada, 2008; Maren et al., 2013). For example, context processing

68 deficits in patients with PTSD can lead to the overgeneralization fear (Grillon and Morgan, 1999;

69 Jovanovic et al., 2012; Kaczkurkin et al., 2017; Lis et al., 2020; Morey et al., 2020, 2015; Orr et

70 al., 2000), deficits in the extinction of fear (Blechert et al., 2007; Jovanovic et al., 2012; Milad et

71 al., 2009; Norrholm et al., 2011; Rougemont-Bücking et al., 2011; Steiger et al., 2015; Wicking et

72 al., 2016), and the renewal of extinguished fear in safe contexts (Garfinkel et al., 2014). This

73 suggests that a complete understanding of how contexts regulate conditioned defensive behavior

74 is essential to identifying neural circuits relevant to fear and anxiety disorders.

Pavlovian fear conditioning has been used for decades to model aversive learning and

76 memory in rodents. Previous work has revealed that contexts can both directly elicit conditioned

77 fear responses and also act as occasion setters to gate the retrieval of aversive memories (Bouton,

78 2002; Fraser and Holland, 2019; Maren et al., 2013). In both cases, fear responses in rodents

79 typically manifest as defensive freezing behavior, which is used as the primary metric of

80 conditioned fear in rodents. Predatory imminence theory posits that defensive behavior scales with

81 threat proximity on a spatiotemporal scale such that freezing behavior is seen in post-encounter

82 modes (once threat has been realized) whereas flight behavior is part of the circa-strike defensive

83 mode (when threat is proximal) (Fanselow and Lester, 1988). This has been demonstrated in both 
84 humans and rodents using naturalistic predator threats (Mobbs et al., 2007; Yilmaz and Meister,

85 2013), but it remains unclear whether conditioned threats (such as auditory conditioned stimuli)

86 can drive circa-strike behavior such as flight. Recently, Fadok and colleagues developed a

87 modified auditory Pavlovian fear conditioning procedure that uses a serial-compound stimulus

88 (SCS) to elicit both freezing and flight defensive modes in mice (Fadok et al., 2017). The SCS is

89 comprised of a pure tone stimulus immediately followed by a white noise stimulus that elicits two

90 conditioned responses (CRs): freezing and flight behavior, respectively. Moreover, they show that

91 the switch between freezing and flight behavior is gated by microcircuitry within the central

92 nucleus of the amygdala (CeA), a structure critical to the expression of Pavlovian CRs (Janak and

93 Tye, 2015; Killcross et al., 1997). Interestingly, in the SCS paradigm flight behavior is normally

94 limited to the conditioning chamber and freezing dominates when the SCS is presented in a

95 different context. This procedure presents a unique opportunity to investigate mechanisms by

96 which context and associative memory may scale between freezing and flight defensive modes.

97 In order to fully reveal the neural circuits that may underly pathological fear, we must

98 understand the distinct neural circuits that underly various defensive modes and how they may be

99 gated or modulated by context (Mobbs et al., 2020). Here we sought to determine the behavioral

100 and neural mechanisms mediating the influence of context on the expression of defensive

101 behaviors to an SCS. One possibility is that context serves as an occasion setter and promotes SCS-

102 evoked flight behavior in the conditioning context, but not in other contexts. Another possibility

103 is that direct context-US associations produce fear that summates with that to the SCS to elevate

104 threat imminence thereby yielding flight. The occasion setting hypothesis predicts that flight would

105 be specific to the conditioning context and would not be expressed elsewhere, whereas the

106 summation hypothesis predicts that flight would be evoked in any shock-associated context, 
107 regardless of whether it had hosted SCS-shock trials. In a series of experiments to test these

108 competing hypotheses, we found that rats displayed flight behavior when the SCS was presented

109 in a US-associated context different than the conditioning context. Moreover, extinguishing fear

110 to the conditioning context suppressed flight behavior in that context. We further provide evidence

111 that SCS-evoked flight is a conditioned response by showing that flight-like behavior cannot be

112 explained by sensitization or fear-potentiated startle. Finally, we show that pharmacologically

113 inactivating either the CeA or BNST, brain regions that are critical to the expression of contextual

114 fear, reduces flight-like behavior. We thus argue that SCS-evoked flight behavior is a high fear

115 state driven by the summation of cued, contextual, and innate fear.

\section{RESULTS}

118 A conditioned serial-compound stimulus evokes flight behaviors in rats.

119 Previous work shows that SCSs can evoke flight behavior in mice, but it is unknown if this

120 behavior occurs in rats. Therefore, we first sought to determine if rats show flight-like behavior to

121 an SCS using the behavioral protocol first described by Fadok and colleagues (Fadok et al., 2017).

122 In this procedure (Figure 1A), rats were first habituated to four SCS presentations (tone $\rightarrow$ white

123 noise; each stimulus consisted of $10 \mathrm{sec}$ trains of $500 \mathrm{~ms}$ pips with an inter-pip interval of $500 \mathrm{~ms}$ )

124 in context A (Day 1), then conditioned with five SCS-US presentations for the next three days in

125 context B (Days 2-4), and finally tested with four SCS-alone presentations in both context A and

126 B (Days 5 and 6; counterbalanced). For this experiment, we quantified 1) freezing as a percentage

127 of time, 2) average motor activity, as well as 3) the number of jumps (all four paws leaving the

128 floor) and darts (rapid movement from one position to another) during both tone and noise

129 components of the SCS. Freezing and activity were quantified automatically 
A
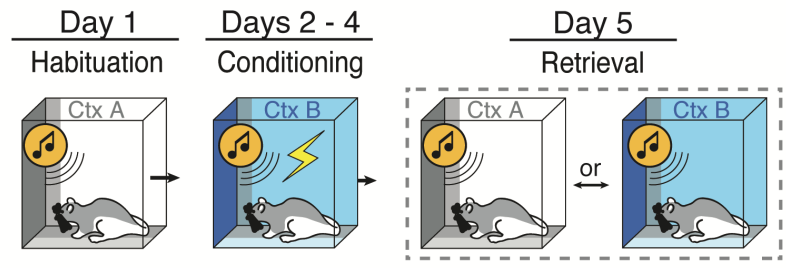

B Serial-Compound

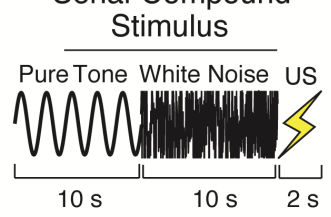

C Habituation
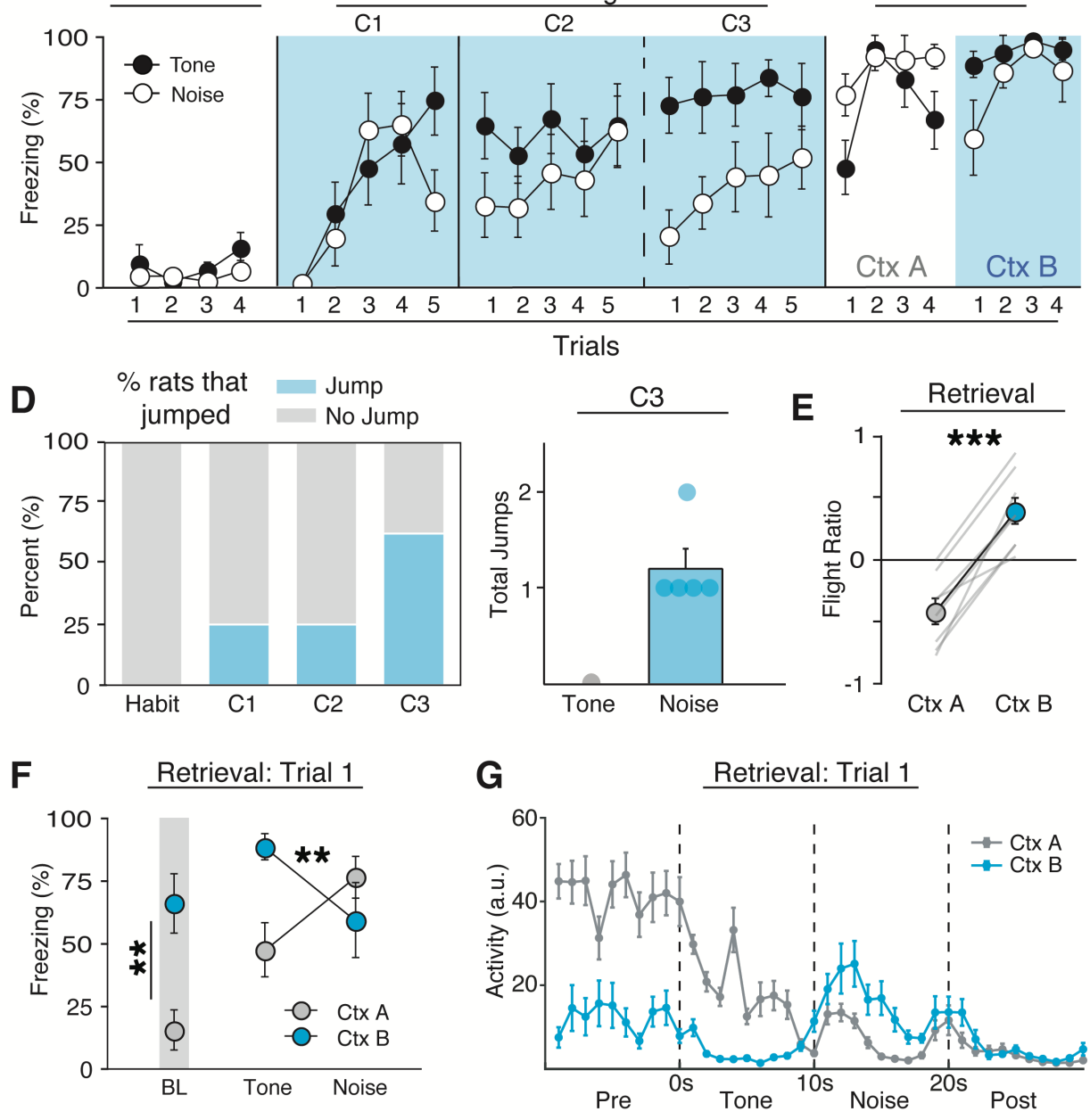

$\mathbf{F}$

Figure 1. Rats display context-dependent flight-like behavior to a conditioned SCS. A, Schematic of the behavioral design used in Experiment 1. B, Schematic representation of the serial-compound stimulus (SCS). C, Average freezing data for tone and noise stimuli during each SCS presentation during Habituation, Conditioning, and Retrieval. Rats showed lower freezing to the noise on the second and third day of conditioning. D, Percentages of rats that showed at least one jump during an SCS for each respective day of behavioral testing. Of the rats that showed at least one jump, jumps were exclusive to noise stimuli (C3). E, Average flight ratio in which positive numbers represent increased movement to the noise relative to tone, whereas negative numbers represent decrease activity relative to tone. Rats displayed flight like behavior when tested in the conditioning, but not habituation context. F, Average freezing data during Retrieval shows that rats tested in the conditioning context showed high freezing during baseline and the first tone presentation but decrease to the noise, whereas rats tested in the habituation context showed low freezing to baseline and the tone which increase to the noise. G, Averaged motor activity data from 10s before SCS onset to 10s after SCS offset in both the Habituation (Ctx A) and Conditioning (Ctx B) contexts. All data are represented as mean $\pm \mathrm{SEM} ;{ }^{*},{ }^{* *}$, and ${ }^{* * *}$ denotes $p<.05, p<.01$, and $p<.001$, respectively. 
144 online by digitizing voltages emitted by force transducers under each chamber; jumps and darts

145 were scored offline from video recordings of the sessions by observer's blind to the experimental

146 conditions.

As shown in Figure 1C, prior to conditioning, SCS presentations produced low levels of

148 freezing and there was no difference in stimulus type on either freezing $[F(1,6)=1.31, p=.295]$

149 or activity $[F(1,6)=2.57, p=.160]$. All rats showed increased freezing behavior throughout the

150 first day of conditioning [main effect of trial, $F(4,24)=8.94, p<.0001]$. Although there was no

151 main effect of stimulus type $[F(1,6)=1.91, p=2.16]$, there was a trial $\mathrm{x}$ stimulus type interaction

$152[F(4,24)=3.33, p=.026]$ with noise producing a decrease in freezing relative to the tone stimulus

153 on the last trial of the first conditioning session (Figure 1C). This suggested that noise onset was

154 associated with a reduction in freezing. Indeed, on the second day of conditioning rats showed less

155 freezing to the noise $\operatorname{CS}[F(1,6)=10.12, p=.019]$, which was mirrored by an increase in activity

$156[F(1,6)=15.27, p=.008]$. Although the rats displayed a clear switch in defensive behavior upon

157 noise onset, the number of jumps to the noise were low with only $\sim 25 \%$ of rats displaying at least

158 one jump (Figure 1D). During this third and final conditioning session, all rats showed an even

159 greater decrease in freezing and increase in activity upon noise onset [Freezing: main effect of

160 stimulus type, $F(1,6)=52.23, p=.0004$; Activity: main effect of stimulus type, $F(1,24)=41.15$,

$161 p=.001]$, and $\sim 60 \%$ of the rats performed at least one jump. Importantly, jumps were nearly

162 exclusive to noise presentations, with only three total jumps observed during tone presentations

163 across all three days of conditioning. Despite a clear increase in activity, rats emitted only a small

164 number of total jumps with only one rat jumping multiple times during the third conditioning

165 session (Figure 1D). 
Recent reports have noted that aversive CSs can elicit darting behavior, particularly in

167 female rats (Gruene et al., 2015). However, we seldomly observed darting behavior to the SCS in

168 male or female rats ( 3 or less total darts across all animals each day). Moreover, there were no sex

169 differences in freezing [main effect: $F(1,6)=.769, p=.414$ ] or activity [main effect: $F(1,6)$

$170=.598, p=.469$ ] evoked by the SCS across conditioning. In summary, a conditioned SCS elicits a

171 clear switch from freezing to activity with infrequent jumps in both male and female rats. It appears

172 that an increase in motor activity (and decrease in freezing) is the dominant mode of SCS-evoked

173 flight-like behavior in rats, compared to frequent jumping previously observed in mice (Fadok et

174 al., 2017; Hersman et al., 2020). We will therefore use white noise-evoked decreases in freezing

175 from here on as the primary metric for flight behavior in rats.

176

177 SCS-evoked flight behavior in rats is context-dependent.

178 Next, we sought to determine if flight behavior in rats is context-dependent as previously shown

179 in mice (Fadok et al., 2017). In previous work, flight behavior was observed in the conditioning

180 context, but not when the SCS was presented in the habituation context. To test this, conditioned

181 rats were placed into either the habituation or conditioning context ( $\mathrm{Ctx} \mathrm{A}$ and $\mathrm{B}$, respectively)

182 and presented the SCS four times without the US. Although there was no difference in overall

183 freezing between contexts $[F(1,6)=.95, p=.367]$ or stimulus type $[F(1,6)=.14, p=.720]$, rats

184 displayed a clear decrease in freezing upon noise onset in the conditioning context (Figure 1C \&

185 F), whereas they instead froze more to the noise presentation in the habituation context [context X

186 stimulus type interaction, $F(1,6)=17.82, p=.006]$. This was again mirrored by activity levels

$187[F(1,6)=14.12, p=.009]$. In other words, rats displayed an increase in activity upon noise onset

188 in the conditioning context, but not the habituation context (Figure 1G). There was no main effect 
189 of sex for either freezing $[F(1,6)=.434, p=.535]$ or activity levels $[F(1,6)=.770, p=.414]$.

190 Interestingly, flight only occurred during the first trial of retrieval testing and rats froze at high

191 levels for the remainder of the test trials [main effect of trial, $F(3,18)=8.25, p=.001]$. This is

192 reminiscent of the rapid extinction of flight behavior previously reported in mice (Fadok et al., 193 2017).

194 Figure 1G shows that when tested in the conditioning context rats exhibited low levels of 195 activity to the tone but increased their activity upon white noise onset. However, in the habituation 196 context, rats exhibited low levels of activity throughout the duration of the SCS. To further 197 quantify this, we computed a "flight ratio", which was the ratio of the difference of noise and tone 198 load-cell activity to the sum of noise and tone load-cell activity for the first retrieval test trial 199 (further described in the methods). This metric spans a scale from -1 to 1 whereby increased 200 activity during noise relative to tone is represented as positive values and decreased relative 201 activity is represented as negative values. As shown in Figure 1E, flight ratios were greater to the 202 noise compared to the tone in the conditioning context relative to the habituation context $[F(1,6)$ $203=86.26, p<.0001]$. There was once again no main effect of $\operatorname{sex}[F(1,6)=.041, p=.911]$. This 204 shows that the SCS-driven flight behavior observed in rats is limited to the conditioning context. 205 Collectively, these results demonstrate that a conditioned SCS drives flight-like behavior in rats 206 manifest as a reduction in freezing punctuated by infrequent jumping behavior, and this pattern of 207 responding to the SCS was context-dependent, as has previously been reported in mice.

209 Flight-like behavior depends on context-US associations.

210 We next investigated what properties of the test context gates flight behavior. One possibility is 211 that context serves as an occasion setter, informing the animal about the SCS-US association in 
212 the conditioning context. Alternatively, flight may be driven by a high fear state resulting from

213 the summation of SCS-US and context-US associations. To discriminate among these possibilities,

214 we explored whether conditioned flight would be expressed in an excitatory context that had never

215 hosted SCS-US trials (i.e., a context in which animals experienced unsignaled shocks).

$\frac{\text { A Day } 1}{\text { Habituation }} \frac{\text { Days } 2-4}{\text { Conditioning }}$
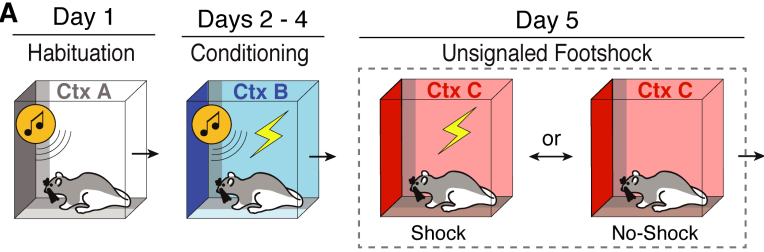

B

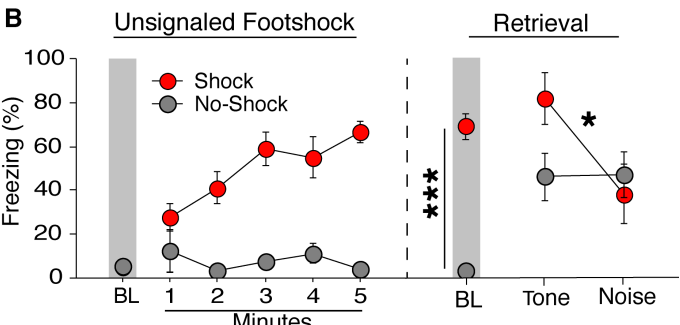

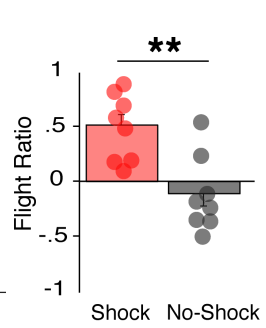

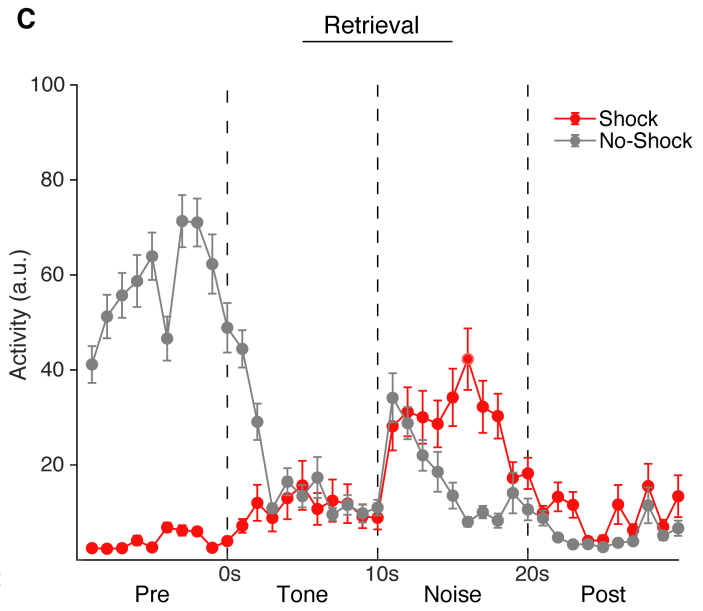

Figure 2. Flight-like behavior depends on context-US associations. A, Behavioral design for Experiment 2. B, Average freezing data shows rats that received unsignaled footshocks (Shock) froze at high levels, whereas rats that were merely exposed to the context (No-Shock) froze at low levels. For Retrieval testing, Shock animals showed higher baseline freezing and a decrease in freezing upon white noise onset whereas No-Shock animals showed low baseline levels and remained freezing at moderate levels throughout the SCS. C, Average flight ratio shows that rats that Shock animals showed a bigger flight response than No-Shock animals. D, Averaged activity data during the first trial of Retrieval for Shock and No-Shock animals. All data are represented as mean \pm SEM; *, **, and *** denotes $p<.05, p<.01$, and $p<.001$, respectively.

To this end, rats first underwent habituation and conditioning as previously described

(Figure 2A). There was once again very little freezing to the SCS prior to conditioning and no difference in stimulus type $[F(1,12)=1.607, p=.229]$. Rats displayed increased freezing across conditioning sessions [main effect of day: $F(2,24)=9.809, p=.0008$ ] as well as noise-elicited

231 decreases in freezing [day X stimulus type interaction: $F(1,12)=14.109, p<.0001$ ]. Next, to test

232 if flight depends on a context-US association, rats were separated into two groups that would either 
234 context for an equal amount of time (Figure 2A, Day 5). As shown in Figure 2B, rats that received

235 unsignaled USs (Shock group) showed increased freezing across the session, whereas rats that 236 were not shocked (No-Shock group) froze at low levels [minutes X group interaction: $F(5,60)=$ $23711.364, p<.0001]$. For retrieval testing, all rats were placed back into context $\mathrm{C}$ and after a 3-minute baseline

239 period were presented one SCS-alone trial. Shock animals showed much higher levels of fear to 240 the context via freezing during the baseline period compared to No-Shock animals $[F(1,12)=$ 241 107.324, $p<.0001]$. Upon SCS presentation, Shock animals displayed a dramatic switch from

242 freezing to activity upon noise onset (Figure 2C), whereas No-Shock animals decreased freezing 243 momentarily, but quickly reverted back to freezing [group X stimulus type interaction: $F(1,14)=$ $2445.928, p=.0289]$. This was mirrored by the flight ratio $[F(1,12)=12.926, p=.0037]$ (Figure 2B).

245 In other words, conditioned animals presented the SCS in a shock-associated context displayed 246 flight-like behavior, whereas animals tested in a neutral context did not. No sex differences were 247 seen during retrieval $[F(1,12)=.498, p=.494]$ nor at any other point in this experiment. Thus, by 248 showing that flight behavior can be evoked in a shock-associated context different from the 249 original conditioning context, these results demonstrate SCS-driven flight-like behavior depends 250 on context-US associations rather than occasion setting by the conditioning context. This suggests 251 that flight to an SCS is driven by a high fear state gated by summation of SCS-US and context-US 252 association.

254 Extinguishing contextual fear reduces flight-like behavior.

255 If contextual fear drives flight to an SCS, then extinguishing that fear should reduce flight 256 behavior. To test this, we habituated and conditioned rats as previously described (Figure 3A), 

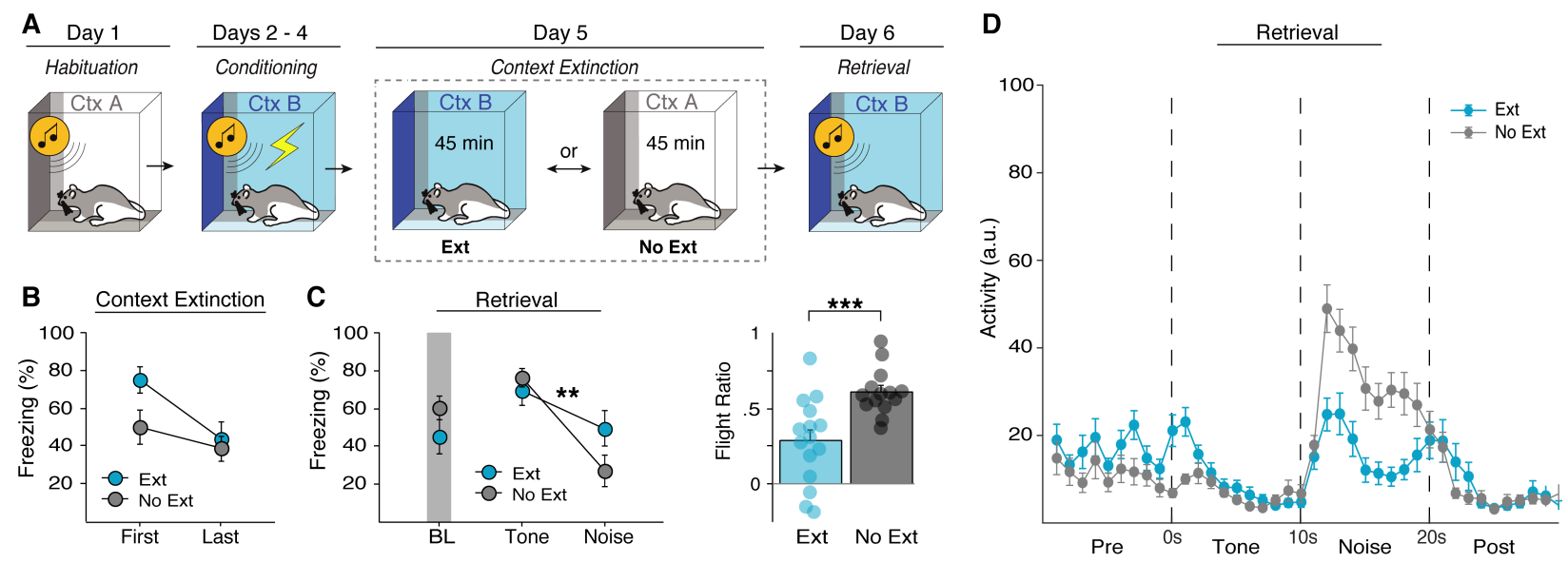

Figure 3. Extinguishing contextual fear reduces flight-like behavior. A, behavioral design for Experiment 3. B, Average freezing data showing that rats that underwent context extinction (Ext) froze at high levels at the beginning of extinction which reduced by the end of extinction. Rats that did not undergo extinction (No-Ext) did not show a reduction in freezing from the first to last block of context exposure. C, Ext animals did not show a significant reduction in baseline freezing, but did show a reduced flight response as shown by the reduced flight ratio. D, Averaged activity data showing that Ext animals showed reduced activity during the white noise stimulus compared to No-Ext animals. All data are represented as mean $\pm \mathrm{SEM} ;{ }^{*},{ }^{* *}$, and ${ }^{* * *}$ denotes $p<.05, p<.01$, and $p<.001$, respectively.

and then extinguished the conditioning context prior to retrieval testing. As in the previous experiments all rats showed similarly low levels of freezing to both stimuli prior to conditioning $[F(1,25)=1.674, p=.2075]$, increased freezing across conditioning days [main effect: $F(2,50)=$

effect: $F(1,25)=69.535, p<.0001]$. Of note, female rats in this experiment showed slightly higher levels of freezing during habituation [main effect: $F(1,25)=5.208, p=.0313$ ], but no sex differences were observed across conditioning days $[F(1,25)=.768, p=.3891]$. After fear for $45 \mathrm{~min}(\mathrm{Ext})$ or they were exposed to the habituation context for an equal amount of time extinction $[F(1,27)=2.994, p=.095]$, planned comparisons revealed that Ext animals showed a significant reduction in freezing $[F(1,14)=16.930, p=.0011]$, whereas No-Ext animals showed 
For retrieval testing, all rats were placed back into the conditioning context and presented

280 four SCS-alone trials. Although baseline freezing was similar between groups $[F(1,27)=1.857$,

$281 p=.1843]$, NoExt rats showed a greater reduction in freezing to noise onset relative to Ext animals

282 [stimulus type $\mathrm{X}$ group interaction, $F(1,25)=15.880, p=.0005$ ], which was again mirrored by

283 changes in activity $[F(1,25)=6.995, p=.0139]$, and flight ratio $[F(1,25)=14.212, p=.0009]$

284 (Figure 3C). In other words, context extinction reduced flight-like behavior (Figure 3D). There

285 was no main effect of sex for any of these metrics during retrieval testing $[F(1,25)=1.388, p$

$286=.2498]$. These results provide converging evidence that SCS-driven flight-like behavior is driven

287 by summation of fear to the SCS and conditioning context.

289 Flight-like responses in rats are specific to white noise and not due to sensitization.

290 One outstanding question is whether flight behavior in rats is driven by threat imminence or

291 stimulus salience. Predatory imminence theory posits that defensive responding scales with threat

292 proximity, and thus, white noise may elicit flight in the SCS paradigm because it is temporally

293 proximal to the shock US. However, recent work shows that SCS-elicited flight behavior in mice

294 is driven by stimulus salience, specifically intensity and high frequency components of white noise

295 (Hersman et al., 2020). Indeed, this work shows in mice that flight behavior is specific to white

296 noise regardless of whether it precedes or follows the pure tone component of the SCS. Moreover,

297 Hersman and colleagues show that flight behavior does not require SCS-US pairings, insofar as an

298 unpaired SCS-US procedure also produced flight to the SCS. This suggests that sensitization or

299 pseudoconditioning might contribute to flight to the SCS. We therefore sought to determine

300 whether the temporal order of the stimuli in the SCS influences the emergence of flight behavior,

301 and if flight-like behavior in rats occurs after unpaired SCS-US trials. To test this (Figure 4A), 
after habituation animals underwent either a standard SCS-US procedure described thus far the same SCS that they were conditioned with (either standard or reversed) without US

A
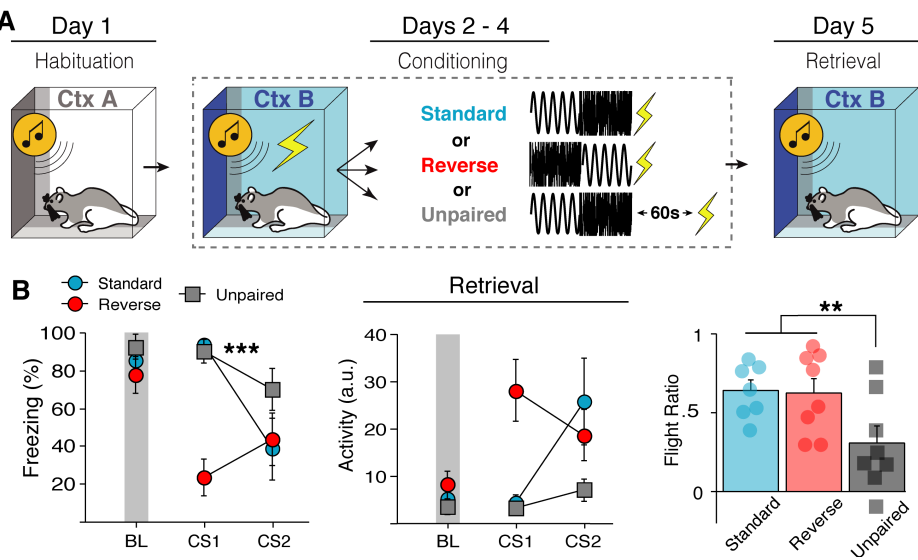
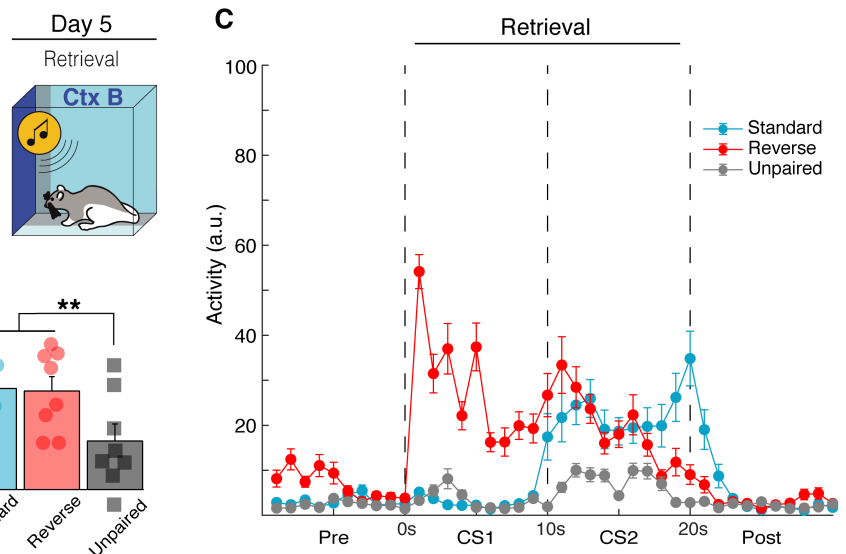

Figure 4. Flight-like responses in rats are specific to white noise and not due to sensitization. A, Behavioral design for Experiment 4. B, Average freezing and activity data showing that flight-like behavior is specific to the white noise stimulus regardless of the temporal of order of the SCS. In a Reversed order SCS, rats show a decrease in freezing and corresponding increase in activity to the first stimuli (noise) rather than the second (tone). The data additionally show that unpairing the SCS and US with a 60-s gap (Unpaired) prevents flight-like behavior compared to Standard SCS-US controls. This all further shown by averaged activity time across time $(\mathbf{C})$. All data are represented as mean $\pm \mathrm{SEM} ; *{ }^{* *}$, and $* * *$ denotes $p<.05, p<.01$, and $p<.001$, respectively.

Prior to conditioning, there was no main effect of either group $[F(2,17)=.858, p=.4416]$, 
in the Reversed group did show increased freezing to tone presentations across habituation which

327 yielded a trial $\mathrm{X}$ sex interaction $[F(3,51)=3.777, p=.0160]$. All animals showed increased

328 freezing across conditioning sessions and all groups showed decreased freezing to the noise

329 relative to the tone, including animals in which the SCS order was reversed [day X stimulus type

$330 \mathrm{X}$ group interaction: $F(4,34)=15.466, p<.0001]$. This suggests that flight responses are indeed

331 specific to white noise, rather than determined by threat proximity. Female rats in this experiment

332 generally showed higher freezing levels across conditioning [main effect of sex: $F(1,17)=5.509$,

$333 p=.0313]$, although no interactions with sex were seen. During retrieval testing, Unpaired animals

334 showed a reduced flight response to white noise [main effect: $F(2,17)=7.646, p=.0043$ ]

335 compared to both Standard $[p=.0103]$ and Reversed groups $[p=.0106]$ (Figure 4B). No main

336 effect of sex was seen $[F(1,17)=1.397, p=.2535]$. In summary, we show here that flight behavior

337 is also exclusive to white noise in rats and suggests that footshock sensitization cannot account for

338 flight behavior. This reaffirms previous findings reported in mice that innately aversive auditory

339 stimuli drive flight response in the SCS paradigm, not threat imminence.

341 An unconditioned SCS fails to evoke flight behavior in a threatening context.

342 As shown in the previous experiment, flight behavior is specific to white noise and appears to be

343 driven by stimulus salience. This raises an alternative possibility that SCS-evoked flight may be

344 nonassociative. White noise is commonly used as an acoustic startle stimulus and it is well known

345 that a startle response can be potentiated when presented in a shock-associated context, a process

346 known as fear-potentiated startle (Davis and Walker, 2014; Luyten et al., 2011; McNish et al.,

347 1997). Although we showed in the last experiment that unpaired SCS/US presentations fail to

348 produce robust flight behavior, an unpaired CS can come to act as a conditioned inhibitor (i.e., 
safety signal) which may have reduced flight behavior (Rescorla, 1969). Thus, we next
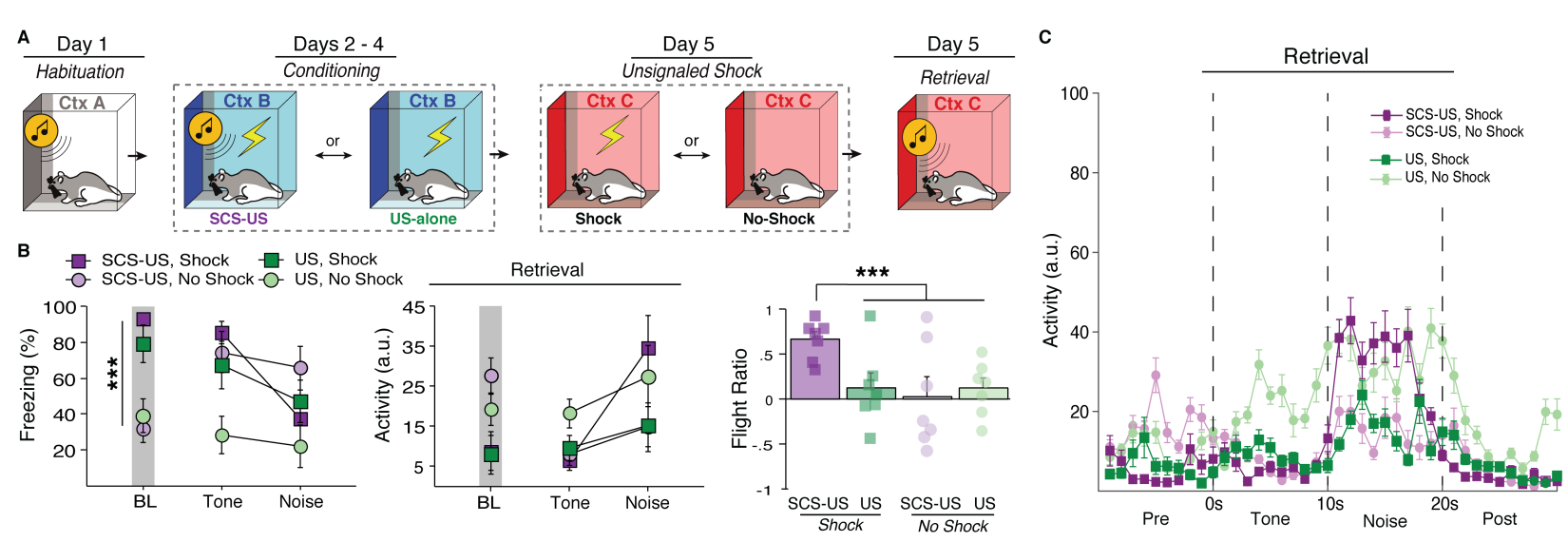

Figure 5. An unconditioned SCS fails to evoke flight behavior in a threatening context. A, Behavioral design for Experiment 5. B, Averaged freezing and activity data showing that rats that received US-alone trials throughout conditioning (US) and tested in a US-associated context (Shock) had a reduced flight response in comparison to rats that received SCS-US pairings. This is shown by increased freezing and decreased activity to the white noise stimulus, and a reduced flight ratio. Rats that were tested in a neutral context (No-Shock) also showed reduced flight responses compared to SCS-US/Shock animals. This is further shown by the average activity trace of each group (D). All data are represented as mean $\pm \mathrm{SEM} ; *, * *$, and $* * *$ denotes $p<.05, p<.01$, and $p<.001$, respectively.

All animals were first habituated with four SCS-alone trials. No differences were seen between

groups $[F(1,24)=.604, p=.445]$, stimulus type $[F(1,24)=3.205, p=.0860]$, or sex $[F(1,24)$

$=.927, p=.4445]$; although females showed slightly higher freezing across habituation trials [trial

X sex interaction: $F(3,72)=2.870, p=.0423]$. To test if SCS driven flight can be explained by

fear-potentiated startle (Figure 5A), animals either underwent standard SCS-US conditioning or

received an equal number of unsignaled USs across three consecutive days. The day after

conditioning, animals were placed into a novel context $\mathrm{C}$ where they either received five US-alone

presentations or context exposure, similar to Experiment 2. Finally, all animals were presented

$\mathrm{SCS}$-alone trials in context $\mathrm{C}$ on the last day of experimentation. This creates a $2 \times 2$ design where 
373 potentiated startle response, we would expect both animals conditioned with SCS-US and US-

374 alone trials to exhibit flight-like behavior in the shock-associated context.

375 During SCS-US conditioning, all animals showed increased freezing across sessions $[F(2$,

$37624)=70.058, p<.0001]$ and lower levels of freezing to noise than tone $[F(1,12)=29.132, p$

$377=.0002$ ], though the difference in freezing between stimuli were similar across days [day $\mathrm{X}$

378 stimuli: $F(2,24)=2.784, p=.0818]$. Animals that received US-alone trials also showed increased

379 freezing across sessions $[F(2,24)=22.404, p<.0001]$. No sex differences were observed during

380 conditioning. One day after conditioning, animals either received five unsignaled footshocks in a

381 novel context $\mathrm{C}$ or were exposed to the context for an equal amount of time. Animals that received

382 footshocks showed an increase in freezing behavior relative to No-Shock animals $[F(5,120)=$

$38310.762, p<.0001]$. Although there was no main effect of $\operatorname{sex}[F(1,24)=.445, p=.5111]$, there

384 was a trial $\mathrm{X}$ group $\mathrm{X}$ sex interaction $[F(35,120)=2.507, p=.0338]$, again driven by slightly

385 higher freezing in female No-Shock animals. On the day of retrieval testing (Figure 5B), Shock

386 animals showed much higher levels of baseline freezing than No-Shock animals $[F(1,23)=$

$38734.997, p<.0001]$ and there was no difference in SCS-US and US-alone groups $[F(1,23)=.145$

$388 p=.7070]$ or sex $[F(1,23)=.064, p=.8025]$. At the first SCS presentation, all groups froze at a

389 high level except for the US-alone/No-Shock group [conditioning X unsignaled shock interaction:

$390 F(1,23)=4.062, p=.0557]$. Upon white noise onset, the SCS-US/Shock group showed a dramatic

391 increase in activity greater than all other groups [flight score main effect: $F(3,24)=3.632, p$

$392=.0272]$, including the US-alone/Shock group $[p=.0193]$ (Figure 5B). Indeed, the US-

393 alone/Shock group showed similar flight-like behavior as animals that were never shocked in that

394 context. We therefore conclude that SCS driven flight behavior does require a direct SCS-US

395 pairing in this paradigm and thus cannot be attributed to sensitization or fear potentiated-startle. 


\section{Muscimol inactivation of the central or extended amygdala attenuates flight behavior}

397 If SCS-evoked flight depends on context-US associations, then inactivating brain regions that are

398 critical for this process should block flight behavior. The bed nucleus of the stria terminalis

399 (BNST) has been shown to be critical for the expression of contextual but not cued fear (Davis et

400 al., 2010; Goode and Maren, 2017; Sullivan et al., 2004), whereas the CeA is critical for both

401 contextual and cued fear (Janak and Tye, 2015; Maren and Quirk, 2004). Based on this, we

402 reasoned that inactivation of the either CeA or BNST would block freezing to the conditioning

403 context and SCS-driven flight responses.

All rats were implanted with cannula targeting either the BNST or CeA one week prior to

SCS habituation and conditioning (Figure 6A-C). During habituation, there were main effects of

stimulus type $[F(1,40)=5.145, p=.029]$ and trial $[F(3,120)=3.284, p=.023]$ with animals

freezing to the SCS across conditioning days $[F(2,80)=117.994, p<.0001]$ with decreased

No sex differences were seen across habituation or conditioning. Immediately prior to retrieval

411 testing rats received local infusions of either the $\mathrm{GABA}_{\mathrm{A}}$ agonist muscimol (MUS) or saline

412 (SAL). Inactivation of either the CeA $[p=.0013]$ or $\mathrm{BNST}[p=.0012]$ reduced baseline freezing

413 relative to SAL controls $[F(1,40)=.901, p=.3482]$, an indication of diminished contextual fear.

414 CeA animals showed lower freezing to the tone [main effect: $F(240)=5.457, p=.008$ ] compared

415 to both SAL $[p=.0028]$ and BNST animals $[p=.038]$ (Figure 6D). Upon noise onset, there was

416 not a stimulus type $\mathrm{X}$ group interaction $[F(1,40)=.901, p=.3482]$, but planned comparisons

417 reveal that SAL animals $[p<.0001]$, but not CeA $[p=.6165]$ or $\operatorname{BNST}[p=.1321]$, showed a

418 reduction in freezing behavior (Figure 6D). This is reflected in the flight ratios [main effect: $F(2$, 
$40)=8.773, p=.0007]$ that show both CeA $[p<.0001]$ and BNST inactivation $[p=.0067]$ blunted

420 flight response (Figure 6G). This can further be seen in averaged activity plots (Figure 6E-F). No

421 sex differences were seen during retrieval. In summary, inactivating either the CeA or BNST was

422 sufficient to block both contextual fear and SCS-evoked flight responses. This provides further

423 evidence that SCS flight is a high fear state gated by a summation of contextual and cued fear.
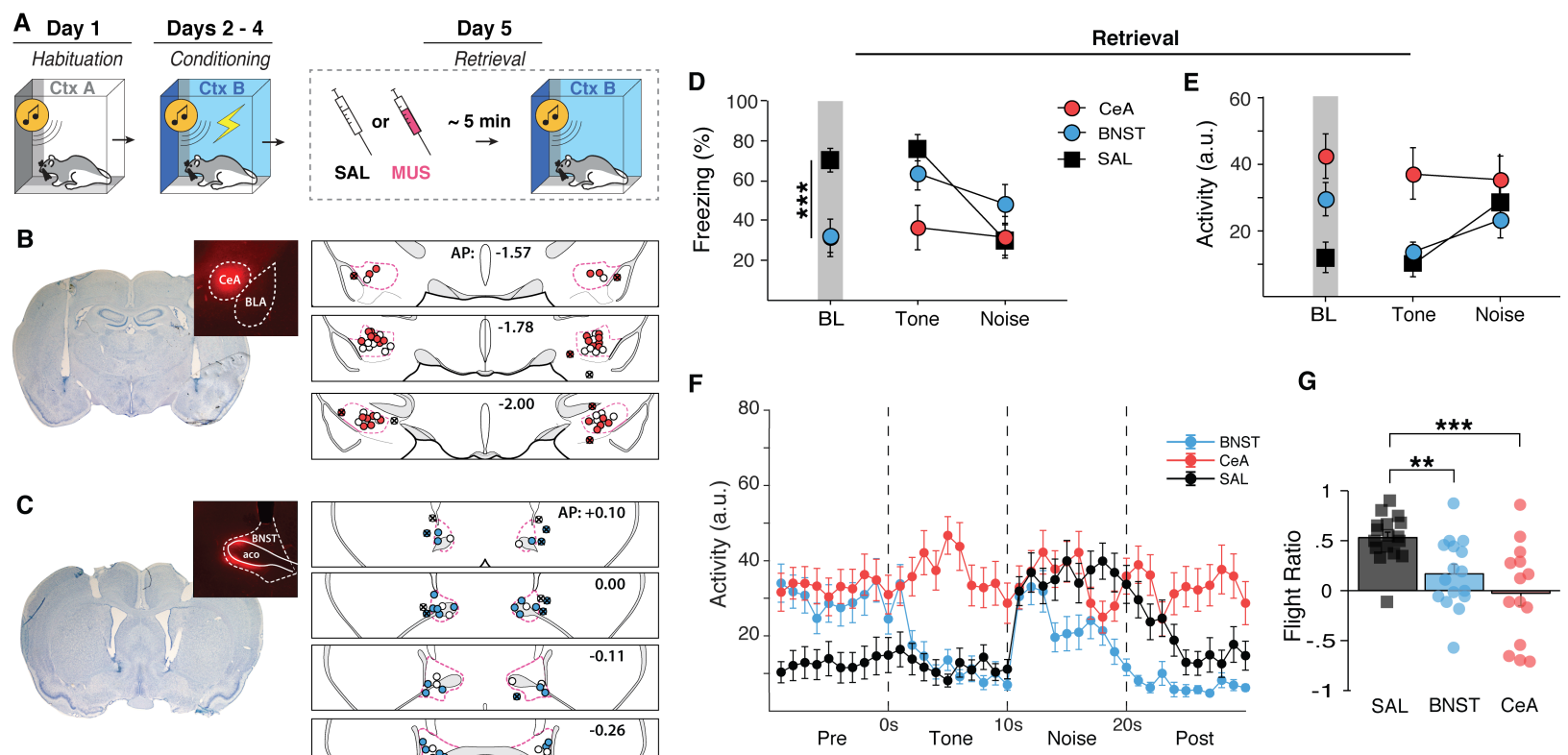

G
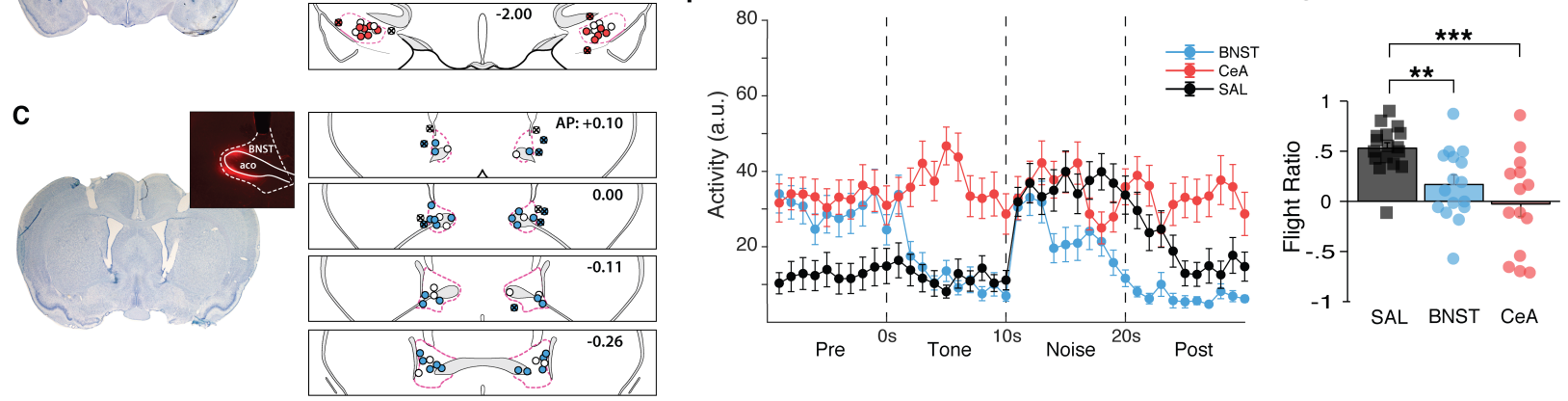

Figure 6. Pharmacological inactivation of either the BNST or CeA disrupts flight-like behavior. A, Behavioral design for Experiment 6. Histological summary of CeA (B) and BNST (C) cannula placements with representative thionin-stained sections and drug spread with fluorescent muscimol. Labeled anterior-posterior coordinates are relative to bregma. D, averaged freezing data showing that CeA and BNST animals both showed reduced baseline freezing. BNST animals increased freezing to tone presentation and remained at higher freezing levels during white noise. CeA animals remained at low levels of freezing during the SCS. E and $\mathbf{F}$, averaged activity levels during the first SCS presentation showing that BNST animals showed a reduced flight response and CeA animals did not increase activity from tone to noise at all despite a lack of freezing behavior. This is reflected in the flight ratio $(\mathbf{G})$. All data are represented as mean $\pm \mathrm{SEM} ;{ }^{*}, * *$, and $* * *$ denotes $p<.05, p<.01$, and $p<.001$, respectively.

\section{DISCUSSION}

438 Here we investigated context-dependent flight behavior evoked by a serial-compound stimulus 
440 behavior can be evoked in rats using the SCS procedure, although jumping was less frequent than

441 previously reported in mice (Fadok et al., 2018). We further found that flight occurs in any shock-

442 associated context and that extinguishing contextual fear to the conditioning chamber suppresses

443 flight-like behavior, demonstrating that SCS-evoked flight behavior reflects the summation of

444 cued and contextual fear. Although flight is specific to white noise, we found that fear-potentiated

445 startle and sensitization could not account for SCS-evoked flight. That is, neither unpaired nor

446 neutral SCS presentations were sufficient to drive flight behavior in a shock-associated context,

447 even when animals had received an equal number of prior footshocks. Finally, we show that

448 pharmacological inactivation of brain regions that are critical for the expression of contextual fear,

449 either the CeA or BNST, is sufficient to block the expression of flight behavior. Together, these

450 data demonstrate that conditioned flight behavior in the SCS paradigm is driven by a high fear

451 state via a combination of contextual and cued fear.

452 Until now only mice had been used to study flight responses in the SCS paradigm and it 453 was unknown if rats would also elicit flight behavior to an SCS (Dong et al., 2019; Fadok et al.,

454 2017; Hersman et al., 2020). We found that the SCS procedure indeed evoked context-dependent

455 flight responses similar to reports in mice, although jumping is less frequent in rats. One important

456 limitation of our study is that we used indirect measures of motion, opposed to other work that

457 used direct measurements of speed via camera which makes direct comparisons of locomotion

458 difficult (Dong et al., 2019; Fadok et al., 2017). Despite this, visual comparison of the increase in

459 activity seen here to those in previous reports appear similar (Dong et al., 2019; Fadok et al., 2017;

460 Hersman et al., 2020). Additionally, increases in activity and jumping seen here were both specific

461 to the white noise stimulus. It is currently unclear why rats display infrequent jumps although we

462 speculate that this may be a species-specific difference. 
Previous work suggests that female rats display more active defensive behaviors, such as defensive darting, in response to an aversive CS (Gruene et al., 2015). Based on this we expected that female rats may be more likely to show flight-like behavior compared to males in the SCS

466 paradigm. However, we did not observe significant sex differences in SCS-evoked jumping or

467 activity levels. Darting behavior was seldom observed during SCS presentations in both female

468 and male rats. This could be due to scoring differences as original reports used an automated 469 detection method (Gruene et al., 2015). Conversely, other work shows that female mice actually 470 exhibit increased freezing in the SCS paradigm, though the average speed of male and female mice 471 during the SCS did not differ (Borkar et al., 2020). We do report here that female rats frequently 472 show increased freezing during SCS habituation and conditioning, although the effects were small 473 and not always present across experiments. Assessing divergent defensive strategies in male and 474 female rats may require machine learning-based behavioral scoring methods (Mathis et al., 2018; 475 Pereira et al., 2020).

As others have shown (Hersman et al., 2020), we found that rats display flight behavior 477 specifically to white noise, even when the order of the SCS is reversed. We further show that flight 478 is not due to sensitization and that flight cannot be explained by fear potentiated startle. This 479 supports previous work demonstrating that stimulus salience determines flight behavior in mice 480 (Hersman et al., 2020). This previous work specifically shows that it is the high-frequency 481 component and intensity ( $>80 \mathrm{dBs}$ ) of white noise that evoke flight. Indeed, loud, high-frequency 482 stimuli appear to innately produce flight behavior in mice. However, it was also shown that 483 sensitization by previous US presentations actually reduces the frequency of flight behavior due 484 to increased competition with freezing behavior (Mongeau et al., 2003). So, how do we reconcile 485 that a direct SCS-US association is necessary to drive flight behavior to a white noise stimulus in 
rats? We believe that the most parsimonious explanation is that flight in the SCS paradigm is driven by a high fear state in which a threshold is reached such that a freeze-to-flight transition occurs. Specifically, SCS flight is driven by the summation of cued, contextual, and innate fear; although all three are not always necessary to elicit flight behavior. For example, flight in mice can be evoked innately to loud, high-frequency stimuli (i.e., without conditioned fear) (Mongeau et al., 2003). Additionally, flight to an SCS can be evoked without a salient high-frequency component by increasing the intensity above 90 dBs (Hersman et al., 2020). In the SCS paradigm, auditory stimuli are presented at 75-80 $\mathrm{dB}$ which appears to be just below the threshold to innately evoke flight responses to white noise (Fadok et al., 2017; Hersman et al., 2020). Coupled with our findings that SCS flight requires both SCS-US and context-US associations, we propose that cued and contextual fear act in sum with stimulus salience to cause a freeze-to-flight transition in the SCS paradigm. or BNST is sufficient to disrupt not only contextual fear, but also context-dependent flight responses in the SCS paradigm. The finding that CeA inactivation disrupted both contextual and

501 cued fear is supported by decades of work demonstrating that the CeA is critical to the expression

502 of conditioned responses (Janak and Tye, 2015; Killcross et al., 1997; Ressler et al., 2020).

503 Moreover, our finding that inactivating the BNST disrupted defensive freezing to the conditioning

504 context, but not the SCS, is in line with previous work showing that the BNST mediates fear to 505 unpredictable threats (Davis et al., 2010; Goode et al., 2019, 2015; Goode and Maren, 2017; 506 Resstel et al., 2008; Sullivan et al., 2004; Walker et al., 2009; Zimmerman and Maren, 2011). In 507 the original report by Fadok and colleagues, they found that SCS-elicited flight is gated by neurons 508 in the $\mathrm{CeA}$ expressing corticotrophin-releasing hormone $(\mathrm{CRH}+)$ that inhibit somatostatin- 
expressing neurons (SOM+) (Fadok et al., 2017). Stimulation of SOM+ neurons can elicit freezing

510 behavior in naïve animals (Li et al., 2013; Penzo et al., 2015, 2014; Yu et al., 2016), however,

511 flight responses evoked by stimulation of CRH+ requires prior conditioning (Fadok et al., 2017).

512 This raises the possibility that SCS-evoked flight behavior may be evoked indirectly via the

513 inhibition of CeA-driven freezing behavior, and thus, may not directly require the CeA. We extend

514 this literature by showing that the CeA is indeed required for SCS-evoked flight behavior by

515 showing that reversible inactivation CeA attenuates flight responses. Collectively, this findings

516 detail for this first time how conditioned auditory and contextual fear may sum with stimulus

517 salience to drive circa-strike behavior.

Considering this, what neural structure may then be responsible for the proposed threshold-

519 like mechanism gating flight behavior? The periaqueductal grey (PAG) is a midbrain structure that

520 is critical to defensive responding downstream of both the CeA and BNST (Tovote et al., 2016,

5212015 ) and appears to have a specialized role in mediating escape behavior (Lefler et al., 2020).

522 Functional differences in the PAGs dorsal-ventral axis exists such that the ventral PAG mediates

523 freezing behavior and the dorsal PAG mediates flight behavior (Assareh et al., 2016; Carrive,

524 1993; Franklin, 2019; Vianna et al., 2001). For example, stimulation of the dorsal PAG can result

525 in both flight and freezing behavior, whereas stimulation of the ventral PAG results exclusively in

526 freezing (Assareh et al., 2016; Carrive, 1993; Chou et al., 2018; Kim et al., 2013; Vianna et al.,

527 2001). Importantly, recent work in mice has indeed shown that the dorsal PAG performs a synaptic

528 threshold mechanism for computing escape behavior in a looming-disc paradigm (Evans et al.,

529 2018). Based on this, we speculate that concurrent CeA and BNST input to the PAG could drive

530 the threshold-like mechanism underlying context-dependent flight behavior (Nagy and Paré,

531 2008). Alternatively, BNST projections to the CeA may represent necessary inputs to drive CeA 
$532 \mathrm{CRH}+$ neurons to gate flight behavior (Fadok et al., 2017; Gungor et al., 2015; Gungor and Paré,

533 2016; Yamauchi et al., 2018). Future work should investigate these pathways and their potential

534 role in mediating flight behavior in the SCS paradigm.

535 To summarize, we have shown that rats display flight-behavior in the SCS paradigm

536 similarly to mice, although rats show less frequent escape-like behaviors such as jumping and

537 darting. Flight-like behavior evoked by the SCS is specific to white noise, gated by contextual fear,

538 and cannot be accounted for by sensitization or fear potentiated startle. We conclude that SCS

539 conditioning results in a high fear state driven by the summation of cued, contextual, and innate

540 fear that drives a freeze-to-flight transition. Future work should investigate the neural mechanisms

541 underlying the transition from post-encounter to circa-strike defensive behaviors and how this is

542 driven by a combination of conditioned and innately aversive stimuli (Fanselow and Lester, 1988;

543 Mobbs et al., 2020). This may reveal important clinical implications for psychiatric disorders that

544 are characterized by high fear states and the dysregulation of contextual processing, such as panic

545 disorder and PTSD (Goddard, 2017; Maren et al., 2013).

\section{MATERIALS AND METHODS}

548 Subjects. Experiments used adult Long-Evans rats $(n=163)$ acquired from Envigo (Indianapolis,

549 IN; 200-240 g upon arrival). Males and females were used in equal numbers throughout all

550 experiments. All animals were housed in a climate-controlled vivarium and kept on a fixed

551 light/dark cycle (lights on starting at 7:00 AM and off at 9:00 PM; experiments took place during

552 the light phase of the cycle). Rats were individually housed in clear plastic cages (with bedding

553 consisting of wood shavings; changed weekly) on a rotating cage rack. Group assignments for

554 behavioral testing was randomized for cage position on the racks. Animals had access to standard 
555 rodent chow and water ad libitum. Animals were handled by the experimenter(s) ( $\sim 30 \mathrm{sec} / \mathrm{day})$ for

556 five consecutive days prior to the start of any surgeries or behavior. All procedures were in

557 accordance with the US National Institutes of Health (NIH) Guide for the Care and Use of

558 Laboratory Animals and were approved by the Texas A\&M University Institutional Animal Care

559 and Use Committee.

561 Apparatuses. All behavioral testing occurred within one of two rooms in the laboratory. Each

562 behavioral room housed eight identical rodent conditioning chambers $(30 \mathrm{~cm} \times 24 \mathrm{~cm} \times 21 \mathrm{~cm}$;

563 MED Associates, Inc.). Each chamber was housed in a larger, external sound-attenuating cabinet.

564 Rear walls, ceilings, and the front doors of the testing chambers were made of Plexiglas, while

565 their sidewalls were made of aluminum. Grid floors of the chambers were comprised of nineteen

566 stainless steel bars (4 mm in diameter) and spaced $1.5 \mathrm{~cm}$ apart (center to center). The grid floors

567 were attached to an electric shock source and a solid-state grid scrambler for delivery of the US

568 (MED Associates, Inc.). A speaker attached to each chamber was used to deliver the auditory CS.

569 As needed for each context, the chambers were equipped with $15 \mathrm{~W}$ house lights, and small fans

570 were embedded in the cabinets (providing background noise of $\sim 70 \mathrm{~dB}$ ). An aluminum pan was

571 inserted beneath the grid floor to collect animal waste. A small camera was attached to the top of

572 the cabinet for video monitoring of behavior.

573 Measurements of freezing and motor activity were performed using an automated system

574 (Maren, 1998). Specifically, each behavioral testing chamber rested on a load-cell platform that

575 was sensitive to cage displacement due to each animal's movements. During behavioral testing,

576 load-cell activity values (ranging from -10 to $+10 \mathrm{~V}$ ) were collected and digitized at $5 \mathrm{~Hz}$ using

577 Threshold Activity Software (MED Associates, Inc.). Offline conversions of the load-cell activity 
578 values were performed to generate absolute values ranging from 0 to 100; lower values indicate

579 minimal cage displacement, which coincided with freezing behaviors in the chambers.

580 Accordingly, freezing bouts were defined as absolute values of $\leq 10$ for $1 \mathrm{~s}$ or more. The

581 percentage of freezing behavior during the pre-SCS baseline and SCS trials was computed for each

582 behavioral session. Motor activity was analyzed by directly reporting the absolute values generated

583 by the Threshold Activity Software (i.e., larger values indicated more movement in the cage).

584 Jumping and darting behavior were manually scored off-line from video recordings by an

585 experimenter blind to experimental conditionings.

Unique contexts (A, B, and C) were used for various phases of behavior testing. Chamber

assignments were unique to each context and group assignments were counterbalanced across test

588 chambers when possible. For each experiment contexts A and B were assigned to different

589 behavioral testing rooms. For context A, the test chamber and pans beneath the grid floors were

590 wiped down with an ammonium hydroxide solution (1\%). The cage lights were turned off,

591 chamber fans were turned on, and the cabinet doors were left open. Black Plexiglas panels were

592 also placed over the grid floors. The behavioral room was lit with white light (red lights were

593 turned off). Animals were transported to and from the chambers using white plastic transport

594 boxes. For context B, an acetic acid solution (3\%) was used to wipe down and scent the chambers,

595 the cage lights were turned on, the chamber fans were turned off, and the cabinet doors were closed.

596 The behavioral room was lit with dim red light (white room lights were turned off). Rats were

597 transported to and from context B using black plastic transport boxes that included a layer of clean

598 bedding. For context $\mathrm{C}$, an ethanol solution (70\%) was used to wipe down and scent the chambers,

599 the cage lights were turned on, the chamber fans were turned on, and the cabinet doors were open.

600 The behavioral room was lit with white lights (red lights remained off) and rats were transported 
601 to and from context $\mathrm{C}$ in white plastic transport boxes with clean bedding. Testing in context $\mathrm{C}$ was always performed in the same behavioral room as context $\mathrm{A}$.

604 Experimental Design. Overviews of each behavioral experiment are provided in the figures. The 605 auditory serial-compound stimulus (SCS) used for all experiments was comprised of ten $500 \mathrm{~ms}$ 606 pure tone pips $(80 \mathrm{~dB}, 7 \mathrm{kHz})$ presented at a frequency of $1 \mathrm{~Hz}(500 \mathrm{~ms}$ inter-pip intervals, $10 \mathrm{~s}$ 607 total length) and immediately followed by ten $500 \mathrm{~ms}$ white noise pips $(80 \mathrm{~dB}, 1-20 \mathrm{kHz})$ presented 608 at a frequency of $1 \mathrm{~Hz}$ (500 ms inter-pip intervals, 10-s total length). During conditioning the SCS 609 was paired with a mild unconditioned footshock stimulus (US, $1.0 \mathrm{~mA}, 2 \mathrm{sec}$ ), unless noted 610 otherwise. Intertrial-intervals (ITI) were always 60 seconds.

612 Experiment 1: Flight behavior in the conditioning vs habituation contexts.

613 In this experiment we tested if the SCS paradigm could evoke flight-like behavior in rats similar 614 to what has previously been reported in mice. To this end, behavioral testing consisted of a 615 habituation session, three conditioning sessions, and a retrieval session. Habituation sessions 616 consisted of a 3 min pre-SCS baseline period followed by 4 SCS presentations without footshock

617 in context A. Next, all rats underwent conditioning consisting of a 3 min pre-SCS baseline period 618 followed by 5 SCS-US presentations in context B for three consecutive days. Multiple days of 619 conditioning were necessary to drive flight responses (Fadok et al., 2017). Retrieval testing 620 consisted of a 3-min pre-SCS baseline period followed by 4 SCS-alone presentations in either 621 context A (habituation) or context B (conditioning). A within-subject design was used such that 622 half the rats were tested in either the habituation or conditioning context first and later were tested 623 in either the conditioning or habituation context, respectively, the subsequent day (counter- 
624 balanced for test order). There was only one group tested in this experiment using a within-subjects

625 design $(n=8)$. No animals were excluded.

627 Experiment 2: Unsignaled footshock

628 For this experiment we wanted to determine if flight-like behavior could be driven in other shock-

629 associated contexts. Behavioral testing for this experiment consisted of a habituation session

630 (context A), three conditioning sessions (context B), a session where rats either received

631 unsignaled footshocks (context C), and a final between-subjects retrieval session in context C.

632 Habituation and conditioning sessions were identical to Experiment 1. For the unsignaled

633 footshock session, all rats were placed into a novel context $\mathrm{C}$ where half were presented a 3-min

634 pre-stimulus baseline followed by 5 unsignaled footshocks $(1.0 \mathrm{~mA}, 2 \mathrm{sec})$ with 60 -sec ITIs

635 (Shock), and the other half were merely exposed to the context for an equal amount of time (No-

636 Shock). The next day all rats were returned to context $\mathrm{C}$ where they were presented with 1 SCS-

637 alone trial following a 3 min baseline period. The groups were as follows: Shock $(n=8)$ and No-

638 Shock $(n=8)$. No animals were excluded.

641 In this experiment we tested if flight behavior could be diminished by extinguishing contextual

642 fear. Behavioral testing consisted of habituation, three days of conditioning, a context extinction

643 session, and a retrieval session. Habituation and conditioning were identical to previous

644 experiments. For context extinction, half of the rats underwent context extinction by exposing

645 them to the conditioning context (context B) for 45 min for two consecutive days (EXT) while the

646 other half were re-exposed to context A for an equal amount of time (No-EXT). The subsequent 
647 day all animals were placed back into context B where they underwent a retrieval session 648 consisting of a 3-min pre-SCS baseline followed by 4 SCS-alone presentations. Groups were as 649 follows: $\operatorname{EXT}(n=14)$ and No-Ext $(n=15)$. Two EXT $(n=2)$ and one No-EXT $(n=1)$ animals 650 were excluded as statistical outliers (flight ratio $>2$ SDs \pm mean).

Experiment 4: Reverse and unpaired SCS

653 This experiment tested both the temporal order of the SCS and the SCS-US relationship on flight

654 behavioral. Behavioral testing for this experiment was similar to previous experiments in that it 655 consisted of one day of habituation (context A), three days of conditioning (context B), and a 656 retrieval session (context C). However, conditioning consisted of either 5 standard SCS-US 657 pairings (Standard), 5 reversed (noise followed by tone) SCS-US pairings (Reverse), or 5 unpaired 658 presentations with a 60-sec delay between each SCS and US (Unpaired). Following the final day 659 of conditioning, all rats were placed back into context B and presented 4 SCS-alone presentations. 660 Groups are as follows: Standard $(n=8)$, Reverse $(n=8)$, and Unpaired $(n=8)$. No animals were 661 excluded.

\section{Experiment 5: Contextual fear potentiation}

664 For this experiment we wanted to determine if the context dependence of flight behavior could be 665 accounted for by fear potentiated startle. This experiment is designed similar to Experiment 2 666 which consisted of habituation (context A), three days of conditioning (context B), unsignaled 667 footshock (context C), and retrieval (context C). However, following habituation, rats were either 668 conditioned with 5 SCS-US pairings (SCS-US) or received 5 unsignaled footshocks (US-alone) in 669 context B for three consecutive days. After conditioning, each of these groups were placed in a 
670 novel context $\mathrm{C}$ where half received unsignaled footshocks (Shock) and half were merely exposed

671 to the context for the same amount of time (No-Shock), thus creating a $2 \times 2$ design. On the final

672 day, all rats were placed in context C and presented 10 SCS-alone trials after a 3-min baseline.

673 Groups were as follows: SCS-US/Shock $(n=8)$, SCS-US/No-Shock $(n=8)$, US-alone/Shock $(n$

$674=8)$, and US-alone/No-Shock $(n=8)$. No animals were excluded.

677 In Experiment 6 we tested if flight behavior that depended on contextual fear could be blocked by

678 inactivating regions necessary for the expression of context fear. Rats were first chronically

679 implanted with bilateral cannula targeting either the CeA or BNST. One week after surgery, all

680 rats underwent habituation (context A) and three days of conditioning (context B) as previously

681 described. Immediately prior to retrieval testing, rats received $0.3-\mu 1$ microinfusions of either the

$682 \mathrm{GABA}_{\mathrm{A}}$ agonist muscimol $(0.1 \mu \mathrm{g} / \mu \mathrm{l})$ or saline at a rate of $0.3 \mu \mathrm{l} / \mathrm{min}$ and infusions needles stayed

683 in-place for at least 2 minutes post-infusion. They were then placed into transport boxes and moved

684 to the behavioral testing room for retrieval testing took place as previously described with a 3-min

685 baseline period and 4 SCS-alone trials. Groups were as follows: CeA $(n=14)$, BNST $(n=14)$,

$686 \operatorname{SAL}(n=16)$.

688 Surgery. Rats were anesthetized with isoflurane ( $5 \%$ induction, $\sim 2 \%$ maintenance), the top of 689 their heads were shaven, and they were placed in a stereotaxic mount (Kopf Instruments, Tujunga,

690 CA). A small incision was made with a scalpel, fascia lining the skull was scrubbed away with 691 cotton swabs, and the scalp was retracted with forceps. The skull was leveled horizontally before 692 burr holes were drilled above either the BNST or CeA. Four additional holes were made anteriorly 
693 and posteriorly (two each) for skull screws. After skull screws were placed, two stainless-steel

694 cannulas (26 gauge, 8mm; Plastics One) were lowered into either the CeA (target coordinates; ML:

695 4.0, AP: -2.0 , DV: -8.0 ) or the BNST (target coordinates; ML: 1.5, AP: 0.0, DV: -6.5). Cannula

696 targeting the BNST were inserted at a $10^{\circ}$ angle to avoid rupturing the ventricle. Thus, angled

697 coordinates used during stereotaxic surgery targeting the BNST were as follows: ML: 3.13, AP:

698 0.0, DV: -6.19 (ML: medial-lateral, AP: anterior-posterior, DV: dorsal-ventral). All coordinates

699 are in reference to the skull surface at bregma. Cannula were then affixed to the skull with dental

700 acrylic and a stainless-steel dummy (30 gauge, $9 \mathrm{~mm}$; Plastics One) was inserted into the guide

701 cannula. Rats were allowed to recover for $\sim 1$ week after surgery before behavioral testing.

703 Drug microinfusions. The day of retrieval testing rats were placed into 5-gallon white buckets

704 and moved into a room adjacent to the vivarium for microinfusions. Dummy cannula internals

705 were removed and a stainless-steel injector (33 gauge, 9mm; Plastics One) connected to

706 polyethylene tubing was inserted into the guide cannula. Polyethylene tubing was connected to 10-

$707 \mu$ l Hamilton syringes that were mounted in an infusion pump (Kd Scientific). Muscimol was

708 diluted to a concentration of $0.1 \mu \mathrm{g} / \mu \mathrm{l}$ in sterile saline. Infusions were made a rate of $0.3 \mu \mathrm{l} / \mathrm{min}$

709 for $1 \mathrm{~min}$ and the injectors were left in place for $2 \mathrm{~min}$ post-infusion to allow for adequate diffusion.

710 Each infusion was verified by movement of an air bubble that separated the drug or sterile saline

711 from distilled water within the polyethylene tubing. Clean dummy internals were inserted into each

712 guide cannula after infusions. All infusions were made $\sim 5$ min prior to behavioral testing.

714 Histology. Twenty-four hours after retrieval testing animals were sacrificed to confirm cannula 715 placement. Animals were overdosed with sodium pentobarbital (Fatal Plus, $100 \mathrm{mg} / \mathrm{ml}, 0.7 \mathrm{ml}$ ), 
716 transcardially perfused with ice-cold saline and fixed with $10 \%$ physiological formalin. Perfused

717 brains were placed in physiological formalin for $14-24$ hours before being moved to a $30 \%$ sucrose

718 solution for a minimum of three days. After three days, or until brains had sunk in $30 \%$ sucrose,

719 all brains were frozen and sectioned at $-20^{\circ}$ on a cryostat at a thickness of $40 \mu \mathrm{m}$. Sections were

720 mounted onto gelatin subbed slides, thionin stained $(0.25 \%)$ to better visualize cannula placement,

721 cover-slipped with Permount (Fisher Scientific), and then imaged on a wide-field stereoscope.

722 A subset of animals infused with fluorescent muscimol to verify drug spread. These

723 animals were overdosed with sodium pentobarbital and infused with $0.3 \mu$ of fluorescent

724 muscimol (BODIPY TMR-X conjugate; Thermo Fisher Scientific) at a rate of $0.3 \mu 1 / \mathrm{min}$. A rest

725 period of 2 minutes was given post-infusion and then animals were immediately sacrificed. Non-

726 perfused brains were placed in a physiological formalin solution for 14-24 hours before being

727 placed in a 30\% sucrose-formalin solution for a minimum of three days. These brains were also

728 sectioned at $-20^{\circ}$ on a cryostat at a thickness of $40 \mu \mathrm{m}$. Sections were then mounted onto subbed

729 slides, coverslipped with fluoromount (Diagnostic Bio-systems), and imaged on a fluorescent

730 microscope at 10x resolution. Hits were confirmed by verifying that the tip of the infusion needles

731 was within the CeA or BNST. Only animals that had bilaterally confirmed placements were

732 included in statistical analyses. Thus, animals in which the tip of either one or both cannulas were

733 outside of the CeA or BNST were excluded from analyses.

735 Statistical analyses. All freezing and raw threshold data were analyzed offline by custom written

736 Python and MATLAB scripts before eventual statistical testing in Statview software. All data were

737 submitted to repeated or factorial analysis of variance (ANOVA) as described for each experiment.

738 Fisher's protected least significant difference (PLSD) test was used for post hoc comparisons of 
739 group means following a significant omnibus $F$ ratio in the ANOVA ( $\alpha$ was set at 0.05 ). No

740 statistical methods were used to predetermine group sizes (group sizes were selected based on prior

741 work and what is common for the field). Sex was included as a biological variable for all statistical

742 comparisons. Data distributions were assumed to be normal, but these were not formally tested.

743 All data are represented as means \pm S.E.M.

745 ACKNOWLEDGEMENTS

746 Supported by grants from the National Institutes of Health (R01MH065961 and R01MH117852

747 to S.M.).

748

\section{ADDITIONAL INFORMATION}

750 Competing interests

751 The authors declare no competing interests.

752

753 Author contributions

754 M.T. and S.M. designed experiments; M.T., N.W., I.H., K.R., R.R., and C.O. performed the

755 experiments; M.T. and N.W. analyzed data; M.T. and S.M. wrote the manuscript. 


\section{References}

Assareh, N., Sarrami, M., Carrive, P., McNally, G.P., 2016. The organization of defensive behavior elicited by optogenetic excitation of rat lateral or ventrolateral periaqueductal gray. Behav. Neurosci. 130, 406-414. doi:10.1037/bne0000151

Blechert, J., Michael, T., Vriends, N., Margraf, J., Wilhelm, F.H., 2007. Fear conditioning in posttraumatic stress disorder: evidence for delayed extinction of autonomic, experiential, and behavioural responses. Behav. Res. Ther. 45, 2019-2033. doi:10.1016/j.brat.2007.02.012

Borkar, C.D., Dorofeikova, M., Le, Q.-S.E., Vutukuri, R., Vo, C., Hereford, D., Resendez, A., Basavanhalli, S., Sifnugel, N., Fadok, J.P., 2020. Sex differences in behavioral responses during a conditioned flight paradigm. Behav. Brain Res. 389, 112623. doi:10.1016/j.bbr.2020.112623

Bouton, M.E., 2002. Context, ambiguity, and unlearning: sources of relapse after behavioral extinction. Biol. Psychiatry 52, 976-986. doi:10.1016/S0006-3223(02)01546-9

Carrive, P., 1993. The periaqueductal gray and defensive behavior: functional representation and neuronal organization. Behav. Brain Res. 58, 27-47. doi:10.1016/0166-4328(93)90088-8

Chou, X.-L., Wang, X., Zhang, Z.-G., Shen, L., Zingg, B., Huang, J., Zhong, W., Mesik, L., Zhang, L.I., Tao, H.W., 2018. Inhibitory gain modulation of defense behaviors by zona incerta. Nat. Commun. 9, 1151. doi:10.1038/s41467-018-03581-6

Davis, M., Walker, D.L., 2014. Role of bed nucleus of the stria terminalis and amygdala AMPA receptors in the development and expression of context conditioning and sensitization of startle by prior shock. Brain Struct. Funct. 219, 1969-1982. doi:10.1007/s00429-0130616-5

Davis, M., Walker, D.L., Miles, L., Grillon, C., 2010. Phasic vs sustained fear in rats and humans: role of the extended amygdala in fear vs anxiety. Neuropsychopharmacology 35 , 105-135. doi:10.1038/npp.2009.109

Dong, P., Wang, H., Shen, X.-F., Jiang, P., Zhu, X.-T., Li, Y., Gao, J.-H., Lin, S., Huang, Y., He, X.-B., Xu, F.-Q., Duan, S., Lian, H., Wang, H., Chen, J., Li, X.-M., 2019. A novel cortico-intrathalamic circuit for flight behavior. Nat. Neurosci. 22, 941-949. doi:10.1038/s41593-019-0391-6

Evans, D.A., Stempel, A.V., Vale, R., Ruehle, S., Lefler, Y., Branco, T., 2018. A synaptic threshold mechanism for computing escape decisions. Nature 558, 590-594. doi: 10.1038/s41586-018-0244-6

Fadok, J.P., Krabbe, S., Markovic, M., Courtin, J., Xu, C., Massi, L., Botta, P., Bylund, K., Müller, C., Kovacevic, A., Tovote, P., Lüthi, A., 2017. A competitive inhibitory circuit for selection of active and passive fear responses. Nature 542, 96-100. doi:10.1038/nature21047

Fadok, J.P., Markovic, M., Tovote, P., Lüthi, A., 2018. New perspectives on central amygdala function. Curr. Opin. Neurobiol. 49, 141-147. doi:10.1016/j.conb.2018.02.009

Fanselow, M.S., Lester, L.S., 1988. A functional behavioristic approach to aversively motivated behavior: Predatory imminence as a determinant of the topography of defensive behavior.

Franklin, T.B., 2019. Recent advancements surrounding the role of the periaqueductal gray in predators and prey. Front. Behav. Neurosci. 13, 60. doi:10.3389/fnbeh.2019.00060

Fraser, K.M., Holland, P.C., 2019. Occasion setting. Behav. Neurosci. 133, 145-175. doi:10.1037/bne0000306 
Garfinkel, S.N., Abelson, J.L., King, A.P., Sripada, R.K., Wang, X., Gaines, L.M., Liberzon, I., 2014. Impaired contextual modulation of memories in PTSD: an fMRI and psychophysiological study of extinction retention and fear renewal. J. Neurosci. 34, 13435-13443. doi:10.1523/JNEUROSCI.4287-13.2014

Goddard, A.W., 2017. The neurobiology of panic: A chronic stress disorder. Chronic Stress 1, 247054701773603. doi:10.1177/2470547017736038

Goode, T.D., Kim, J.J., Maren, S., 2015. Reversible inactivation of the bed nucleus of the stria terminalis prevents reinstatement but not renewal of extinguished fear. Eneuro 2. doi:10.1523/ENEURO.0037-15.2015

Goode, T.D., Maren, S., 2017. Role of the bed nucleus of the stria terminalis in aversive learning and memory. Learn. Mem. 24, 480-491. doi:10.1101/1m.044206.116

Goode, T.D., Ressler, R.L., Acca, G.M., Miles, O.W., Maren, S., 2019. Bed nucleus of the stria terminalis regulates fear to unpredictable threat signals. Elife 8. doi:10.7554/eLife.46525

Grillon, C., Morgan, C.A., 1999. Fear-potentiated startle conditioning to explicit and contextual cues in Gulf War veterans with posttraumatic stress disorder. J. Abnorm. Psychol. 108, 134-142. doi:10.1037//0021-843x.108.1.134

Gruene, T.M., Flick, K., Stefano, A., Shea, S.D., Shansky, R.M., 2015. Sexually divergent expression of active and passive conditioned fear responses in rats. Elife 4. doi:10.7554/eLife.11352

Gungor, N.Z., Paré, D., 2016. Functional heterogeneity in the bed nucleus of the stria terminalis. J. Neurosci. 36, 8038-8049. doi:10.1523/JNEUROSCI.0856-16.2016

Gungor, N.Z., Yamamoto, R., Paré, D., 2015. Optogenetic study of the projections from the bed nucleus of the stria terminalis to the central amygdala. J. Neurophysiol. 114, 2903-2911. doi:10.1152/jn.00677.2015

Hersman, S., Allen, D., Hashimoto, M., Brito, S.I., Anthony, T.E., 2020. Stimulus salience determines defensive behaviors elicited by aversively conditioned serial compound auditory stimuli. Elife 9. doi:10.7554/eLife.53803

Janak, P.H., Tye, K.M., 2015. From circuits to behaviour in the amygdala. Nature 517, 284-292. doi:10.1038/nature14188

Jovanovic, T., Kazama, A., Bachevalier, J., Davis, M., 2012. Impaired safety signal learning may be a biomarker of PTSD. Neuropharmacology 62, 695-704. doi:10.1016/j.neuropharm.2011.02.023

Kaczkurkin, A.N., Burton, P.C., Chazin, S.M., Manbeck, A.B., Espensen-Sturges, T., Cooper, S.E., Sponheim, S.R., Lissek, S., 2017. Neural substrates of overgeneralized conditioned fear in PTSD. Am. J. Psychiatry 174, 125-134. doi:10.1176/appi.ajp.2016.15121549

Killcross, S., Robbins, T.W., Everitt, B.J., 1997. Different types of fear-conditioned behaviour mediated by separate nuclei within amygdala. Nature 388, 377-380. doi:10.1038/41097

Kim, E.J., Horovitz, O., Pellman, B.A., Tan, L.M., Li, Q., Richter-Levin, G., Kim, J.J., 2013. Dorsal periaqueductal gray-amygdala pathway conveys both innate and learned fear responses in rats. Proc. Natl. Acad. Sci. USA 110, 14795-14800. doi:10.1073/pnas.1310845110

Lefler, Y., Campagner, D., Branco, T., 2020. The role of the periaqueductal gray in escape behavior. Curr. Opin. Neurobiol. 60, 115-121. doi:10.1016/j.conb.2019.11.014

Li, H., Penzo, M.A., Taniguchi, H., Kopec, C.D., Huang, Z.J., Li, B., 2013. Experiencedependent modification of a central amygdala fear circuit. Nat. Neurosci. 16, 332-339. doi:10.1038/nn.3322 
Liberzon, I., Abelson, J.L., 2016. Context Processing and the Neurobiology of Post-Traumatic Stress Disorder. Neuron 92, 14-30. doi:10.1016/j.neuron.2016.09.039

Liberzon, I., Sripada, C.S., 2008. The functional neuroanatomy of PTSD: a critical review. Prog. Brain Res. 167, 151-169. doi:10.1016/S0079-6123(07)67011-3

Lis, S., Thome, J., Kleindienst, N., Mueller-Engelmann, M., Steil, R., Priebe, K., Schmahl, C., Hermans, D., Bohus, M., 2020. Generalization of fear in post-traumatic stress disorder. Psychophysiology 57, e13422. doi:10.1111/psyp.13422

Luyten, L., van Kuyck, K., Vansteenwegen, D., Nuttin, B., 2011. Electrolytic lesions of the bed nucleus of the stria terminalis disrupt freezing and startle potentiation in a conditioned context. Behav. Brain Res. 222, 357-362. doi:10.1016/j.bbr.2011.03.066

Maren, S., 1998. Overtraining does not mitigate contextual fear conditioning deficits produced by neurotoxic lesions of the basolateral amygdala. J. Neurosci. 18, 3088-3097.

Maren, S., Phan, K.L., Liberzon, I., 2013. The contextual brain: implications for fear conditioning, extinction and psychopathology. Nat. Rev. Neurosci. 14, 417-428. doi:10.1038/nrn3492

Maren, S., Quirk, G.J., 2004. Neuronal signalling of fear memory. Nat. Rev. Neurosci. 5, 844852. doi:10.1038/nrn1535

Mathis, A., Mamidanna, P., Cury, K.M., Abe, T., Murthy, V.N., Mathis, M.W., Bethge, M., 2018. DeepLabCut: markerless pose estimation of user-defined body parts with deep learning. Nat. Neurosci. 21, 1281-1289. doi:10.1038/s41593-018-0209-y

McNish, K.A., Gewirtz, J.C., Davis, M., 1997. Evidence of contextual fear after lesions of the hippocampus: a disruption of freezing but not fear-potentiated startle. J. Neurosci. 17, 9353-9360.

Milad, M.R., Pitman, R.K., Ellis, C.B., Gold, A.L., Shin, L.M., Lasko, N.B., Zeidan, M.A., Handwerger, K., Orr, S.P., Rauch, S.L., 2009. Neurobiological basis of failure to recall extinction memory in posttraumatic stress disorder. Biol. Psychiatry 66, 1075-1082. doi:10.1016/j.biopsych.2009.06.026

Mobbs, D., Headley, D.B., Ding, W., Dayan, P., 2020. Space, Time, and Fear: Survival Computations along Defensive Circuits. Trends Cogn. Sci. (Regul. Ed.) 24, 228-241. doi:10.1016/j.tics.2019.12.016

Mobbs, D., Petrovic, P., Marchant, J.L., Hassabis, D., Weiskopf, N., Seymour, B., Dolan, R.J., Frith, C.D., 2007. When fear is near: threat imminence elicits prefrontal-periaqueductal gray shifts in humans. Science 317, 1079-1083. doi:10.1126/science.1144298

Mongeau, R., Miller, G.A., Chiang, E., Anderson, D.J., 2003. Neural correlates of competing fear behaviors evoked by an innately aversive stimulus. J. Neurosci. 23, 3855-3868.

Morey, R.A., Dunsmoor, J.E., Haswell, C.C., Brown, V.M., Vora, A., Weiner, J., Stjepanovic, D., Wagner, H.R., VA Mid-Atlantic MIRECC Workgroup, LaBar, K.S., 2015. Fear learning circuitry is biased toward generalization of fear associations in posttraumatic stress disorder. Transl. Psychiatry 5, e700. doi:10.1038/tp.2015.196

Morey, R.A., Haswell, C.C., Stjepanović, D., Mid-Atlantic MIRECC Workgroup, Dunsmoor, J.E., LaBar, K.S., 2020. Neural correlates of conceptual-level fear generalization in posttraumatic stress disorder. Neuropsychopharmacology 45, 1380-1389. doi:10.1038/s41386-020-0661-8

Nagy, F.Z., Paré, D., 2008. Timing of impulses from the central amygdala and bed nucleus of the stria terminalis to the brain stem. J. Neurophysiol. 100, 3429-3436.

doi:10.1152/jn.90936.2008 
Norrholm, S.D., Jovanovic, T., Olin, I.W., Sands, L.A., Karapanou, I., Bradley, B., Ressler, K.J., 2011. Fear extinction in traumatized civilians with posttraumatic stress disorder: relation to symptom severity. Biol. Psychiatry 69, 556-563. doi:10.1016/j.biopsych.2010.09.013

Orr, S.P., Metzger, L.J., Lasko, N.B., Macklin, M.L., Peri, T., Pitman, R.K., 2000. De novo conditioning in trauma-exposed individuals with and without posttraumatic stress disorder. J. Abnorm. Psychol. 109, 290-298. doi:10.1037//0021-843X.109.2.290

Penzo, M.A., Robert, V., Li, B., 2014. Fear conditioning potentiates synaptic transmission onto long-range projection neurons in the lateral subdivision of central amygdala. J. Neurosci. 34, 2432-2437. doi:10.1523/JNEUROSCI.4166-13.2014

Penzo, M.A., Robert, V., Tucciarone, J., De Bundel, D., Wang, M., Van Aelst, L., Darvas, M., Parada, L.F., Palmiter, R.D., He, M., Huang, Z.J., Li, B., 2015. The paraventricular thalamus controls a central amygdala fear circuit. Nature 519, 455-459. doi:10.1038/nature13978

Pereira, T.D., Shaevitz, J.W., Murthy, M., 2020. Quantifying behavior to understand the brain. Nat. Neurosci. doi:10.1038/s41593-020-00734-z

Rescorla, R.A., 1969. Pavlovian conditioned inhibition. Psychol. Bull. 72, 77-94. doi: $10.1037 / \mathrm{h} 0027760$

Ressler, R.L., Goode, T.D., Evemy, C., Maren, S., 2020. NMDA receptors in the CeA and BNST differentially regulate fear conditioning to predictable and unpredictable threats. Neurobiol. Learn. Mem. 174, 107281. doi:10.1016/j.nlm.2020.107281

Resstel, L.B.M., Alves, F.H.F., Reis, D.G., Crestani, C.C., Corrêa, F.M.A., Guimarães, F.S., 2008. Anxiolytic-like effects induced by acute reversible inactivation of the bed nucleus of stria terminalis. Neuroscience 154, 869-876. doi:10.1016/j.neuroscience.2008.04.007

Rougemont-Bücking, A., Linnman, C., Zeffiro, T.A., Zeidan, M.A., Lebron-Milad, K., Rodriguez-Romaguera, J., Rauch, S.L., Pitman, R.K., Milad, M.R., 2011. Altered processing of contextual information during fear extinction in PTSD: an fMRI study. CNS Neurosci Ther 17, 227-236. doi:10.1111/j.1755-5949.2010.00152.x

Steiger, F., Nees, F., Wicking, M., Lang, S., Flor, H., 2015. Behavioral and central correlates of contextual fear learning and contextual modulation of cued fear in posttraumatic stress disorder. Int. J. Psychophysiol. 98, 584-593. doi:10.1016/j.ijpsycho.2015.06.009

Sullivan, G.M., Apergis, J., Bush, D.E.A., Johnson, L.R., Hou, M., Ledoux, J.E., 2004. Lesions in the bed nucleus of the stria terminalis disrupt corticosterone and freezing responses elicited by a contextual but not by a specific cue-conditioned fear stimulus. Neuroscience 128, 7-14. doi:10.1016/j.neuroscience.2004.06.015

Tovote, P., Esposito, M.S., Botta, P., Chaudun, F., Fadok, J.P., Markovic, M., Wolff, S.B.E., Ramakrishnan, C., Fenno, L., Deisseroth, K., Herry, C., Arber, S., Lüthi, A., 2016. Midbrain circuits for defensive behaviour. Nature 534, 206-212. doi:10.1038/nature 17996

Tovote, P., Fadok, J.P., Lüthi, A., 2015. Neuronal circuits for fear and anxiety. Nat. Rev. Neurosci. 16, 317-331. doi:10.1038/nrn3945

Vianna, D.M., Graeff, F.G., Landeira-Fernandez, J., Brandão, M.L., 2001. Lesion of the ventral periaqueductal gray reduces conditioned fear but does not change freezing induced by stimulation of the dorsal periaqueductal gray. Learn. Mem. 8, 164-169. doi:10.1101/lm.36101

Walker, D.L., Miles, L.A., Davis, M., 2009. Selective participation of the bed nucleus of the stria terminalis and CRF in sustained anxiety-like versus phasic fear-like responses. Prog. 
Neuropsychopharmacol. Biol. Psychiatry 33, 1291-1308.

doi:10.1016/j.pnpbp.2009.06.022

Wicking, M., Steiger, F., Nees, F., Diener, S.J., Grimm, O., Ruttorf, M., Schad, L.R., Winkelmann, T., Wirtz, G., Flor, H., 2016. Deficient fear extinction memory in posttraumatic stress disorder. Neurobiol. Learn. Mem. 136, 116-126. doi:10.1016/j.nlm.2016.09.016

Yamauchi, N., Takahashi, D., Sugimura, Y.K., Kato, F., Amano, T., Minami, M., 2018. Activation of the neural pathway from the dorsolateral bed nucleus of the stria terminalis to the central amygdala induces anxiety-like behaviors. Eur. J. Neurosci. 48, 3052-3061. doi:10.1111/ejn.14165

Yilmaz, M., Meister, M., 2013. Rapid innate defensive responses of mice to looming visual stimuli. Curr. Biol. 23, 2011-2015. doi:10.1016/j.cub.2013.08.015

Yu, K., Garcia da Silva, P., Albeanu, D.F., Li, B., 2016. Central amygdala somatostatin neurons gate passive and active defensive behaviors. J. Neurosci. 36, 6488-6496. doi:10.1523/JNEUROSCI.4419-15.2016

Zimmerman, J.M., Maren, S., 2011. The bed nucleus of the stria terminalis is required for the expression of contextual but not auditory freezing in rats with basolateral amygdala lesions. Neurobiol. Learn. Mem. 95, 199-205. doi:10.1016/j.nlm.2010.11.002 